\title{
ON CONVOLUTION OF BOAS TRANSFORM OF WAVELETS
}

\author{
NIKHIL KHANNA ${ }^{\dagger}$ AND LEENA KATHURIA
}

$\begin{array}{lll}\text { Date of Receiving } & : & 30.03 .2018 \\ \text { Date of Revision } & : & 08.05 .2019 \\ \text { Date of Acceptance } & : & 14.05 .2019\end{array}$

\begin{abstract}
In this paper, we show that the Boas transform of Convolution (crosscorrelation) of two wavelets satisfies the required admissibility and regularity conditions. These resulting new wavelets are then employed to analyze Boas transform of convolved (cross-correlated) signals by means of Boas transform wavelet convolution and cross-correlation theorems. Analogously to Bedrosian theorem, Boas transform product theorem is given.
\end{abstract}

\section{Introduction}

Boas [2] introduced an integral transform associated to the Hilbert transform, which emerged due to the study of the class of functions having Fourier transform, which vanishes on a finite interval. This transform was known by Boas transform, which finds an application in the theory of filters in electrical engineering. A filter is a system, having some frequency selective mechanism. Recall from [30], that the system transfer function of a high pass filter is given by $H(w)= \begin{cases}A e^{i t_{o} w}, & \text { if }|w| \geq 1 \\ 0, & \text { otherwise }\end{cases}$ Thus, any finite energy signal $f$ passing through a high pass filter gives an output $g$ such that $\hat{g}(w)=H(w) \hat{f}(w)$. Thus, $\hat{g}$ vanishes on $(-1,1)$. Using Boas' theorems, one can characterize the output of the high pass filter in two ways: (i) a signal $g$ is the output of a high pass filter if and only if $\mathcal{B}(\mathcal{B} g)=-g$, (ii) if $g$ is an output of high pass filter, then $\mathcal{B} g=\mathcal{H} g$. Boas transform was further studied by Goldberg [8], Heywood [10] and Zaidi [29]. For various details related to Boas transform, one may refer to [30] and see [19] for details on Hilbert transform. The wavelet transform finds a significant

2010 Mathematics Subject Classification. 42A38, 42C40, 44A15, 44A60.

Key words and phrases. Boas transform, wavelets, Hilbert transform, Fourier transform, vanishing moments.

We thank the referee for his/her valuable comments, constructive and useful remarks, which have improved the paper significantly.

Communicated by: K. T. Poumai

${ }^{\dagger}$ Corresponding author. 